\title{
The properties of molecular clouds across the Magellanic System
}

\author{
Norikazu Mizuno \\ Department of Astrophysics, Nagoya University, Furocho Chikusa-ku, Nagoya 464-8602, Japan \\ email: norikazu@a.phys.nagoya-u.ac.jp
}

\begin{abstract}
Most stars form in Giant Molecular Clouds (GMCs) and regulate the evolution of galaxies in various respects. The formed stars affect the surrounding materials strongly via their UV photons, stellar winds, and supernova explosions, which lead to trigger the formation of next-generations of stars in the GMCs. It is therefore crucial to reveal the distribution and properties of GMCs in a galaxy. The Magellanic System is a unique target to make such detailed comprehensive study of GMCs. This is because it is nearby and the LMC is nearly face-on, making it feasible to unambiguously identify associated young objects within GMCs. Recent millimeter and sub-millimeter observations in the Magellanic System have started to reveal the distribution and properties of the individual GMCs in detail and their relation to star formation activities. From the NANTEN CO surveys, three types of GMCs can be classified in terms of star formation activities; Type I is starless, Type II is with H II regions only, and Type III is associated with active star formation indicated by huge $\mathrm{H}$ II regions and young star clusters. The further observations to obtain detailed structure of the GMCs by Mopra and SEST and to search for the dense cores by ASTE and NANTEN2 in higher tansition lines of CO have been carried out with an angular resolution of about 5 to $10 \mathrm{pc}$. These observations revealed that the differences of the physical properties represent an evolutionary sequence of GMCs in terms of density increase leading to star formation. Type I and II GMCs are at the early phase of star formation where density does not yet become high enough to show active star formation, and Type III GMCs represent the later phase where the average density is increased and the GMCs are forming massive stars.
\end{abstract}

Keywords. stars: formation, ISM: clouds, Magellanic Clouds, radio lines: ISM, submillimeter

\section{Introduction}

The Magellanic system including the LMC, SMC, and Bridge is a valuable astrophysical laboratory to study star formation and cloud evolution because of its proximity to the Sun. In particular, the LMC offers the best site because of its unrivaled closeness and of the nearly face-on view to us. Star formation requires a cool and high-density interstellar medium (ISM) in which most of the hydrogen is in molecular form; therefore, studies of distribution and properties of molecular clouds are very important for understanding the star formation process. Emission from the tracer CO molecule has been widely used to estimate the distribution and amount of $\mathrm{H}_{2}$ in the Galaxy and other galaxies.

The SMC and Bridge are also unique targets for understanding the processes of star formation in a galaxy that is extremely different from the Milky Way, its low metallicity of $1 / 5^{\text {th }}-1 / 10^{\text {th }}$ solar, and its low dust-to-gas ratio. Its history of interactions with the Milky Way and LMC, mean that star formation may be predominantly driven by a combination of tidally-induced galaxy-galaxy interactions and shell formation. These conditions approximate those encountered in young, star-forming galaxies at high redshift. In such a low-metal environment, $\mathrm{CO}$ may not trace the whole amount of $\mathrm{H}_{2}$ in the GMC due to deeper CO photodissociation (see contributions by C. Bot; A. Leroy, this 
volume), but stars form within dense parts of the GMCs which can be traced by CO. For a better understanding of the young massive cluster (populous cluster) formation, which is yet poorly understood, we need to reveal the properties of $\mathrm{CO}$ clouds and their relation to star formation.

In this contribution, the global distributions and properties of GMCs at 40 pc scale by the NANTEN CO survey are presented in sections 2 and 3, and the comparisons with star/clustr formation and $\mathrm{H}$ I gas are discussed in sections 4 and 5 . The new results of submillimeter observations with ASTE and NANTEN2 are presented in section 6.

\section{Giant molecular clouds (GMCs) in the Magellanic System}

The LMC. Fig. 1 shows the molecular clouds detected with the 2nd NANTEN CO Survey (Fukui et al. 2008) on an optical image of the LMC. The mass of the molecular gas in total is $\sim 5 \times 10^{6} \mathrm{M}_{\odot}$, if we use a CO luminosity to hydrogen column density conversion factor, the $X_{\mathrm{CO}}$-factor, of $\sim 7 \times 10^{20} \mathrm{~cm}^{-2}\left(\mathrm{~K} \mathrm{~km} \mathrm{~s}^{-1}\right)^{-1}$ (see section 3). The CO distribution of the LMC is found to be clumpy with several large molecular cloud complexes, unlike the H I gas distribution (Kim et al. 1998), which is composed of many filamentary and shell-like structures. The cloud complex south of 30 Doradus is remarkable, stretching in a nearly straight line from north to south, as already noted in the previous CO observations (Cohen et al. 1988; Kutner et al. 1997; Fukui et al. 1999; Mizuno et al. 2001a). The current survey shows that the clouds in this molecular cloud complex, "the molecular ridge" are actually connected to one another by low-density molecular gas, and the recent high-resolution CO survey with Mopra (MAGMA survey; Ott et al. 2008) have confirmed this feature. The arclike distribution of molecular clouds along the south-eastern optical edge of the galaxy (the "CO Arc" in Fukui et al. 1999) is also clearly seen. The current sensitive survey confirms that this CO Arc indeed clearly represents an arclike edge of the molecular gas distribution in this eastern boundary of the LMC. Some have speculated that this feature is due to hydrodynamical collision between the LMC and SMC (Fujimoto \& Noguchi 1990) or ram pressure pileup of gas due to the motion of the LMC through a halo of hot, diffuse gas (de Boer et al. 1998; Kim et al. 1998). Supershells may also be playing a role in the formation of GMCs as in the case of LMC 4 (Yamaguchi et al. 2001a). A comprehensive comparison between supergiant shells and GMCs shows that only about $1 / 3^{\text {rd }}$ of the GMCs are located towards supershells, suggesting the effects of supershells are not predominant (Yamaguchi et al. 2001b). There is neither an excess nor a deficit of $\mathrm{CO}$ associated with the stellar bar, but the bright H II regions are all clearly associated with molecular clouds.

The SMC and Bridge. Fig. 2 shows the GMCs superimposed on a grayscale image made using the 3.6, 4.5, and $8.0 \mu \mathrm{m}$ bands from the IRAC instrument on the Spitzer Space Telescope (Bolatto et al. 2007). The CO map is from NANTEN (Mizuno et al. 2001b). Unlike the LMC, they are not spread throughout the galaxy but appear preferentially on the northern and southern ends of the galaxy. Another grouping is located to the east (left) of the SMC along the H I bridge that connects the LMC and SMC. The dust emission is associated with the molecular gas traced by $\mathrm{CO}$, but appears to be more extended than the CO emission (Rubio et al. 2004; Leroy et al. 2007; Bot et al. 2007). A lower dust content would have an impact on the physical and chemical properties of the gas component, e.g., low shielding enhancing molecular dissociation rates. This has a direct consequence for the chemical state but also for the physical properties of the gas, less extended emission and a higher degree of clumping would be expected.

The first detection of a molecular cloud in the Magellanic Bridge was reported by Muller et al. (2003) based on CO observations towards a IRAS $100 \mu \mathrm{m}$ emission source 


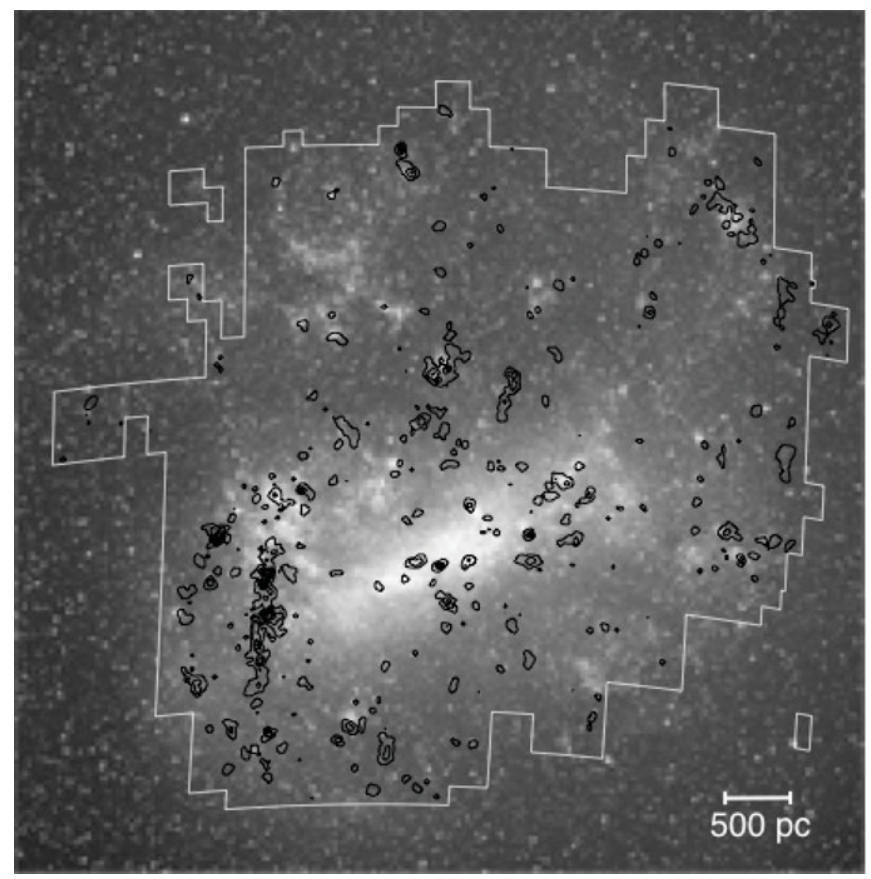

Figure 1. Optical image of the LMC with GMCs mapped with the NANTEN telescope $(\sim 40$ pc resolution) indicated within the boundary of the survey area.

detected in the Bridge as had been the case for SMC CO detections earlier (Rubio et al. 1993). Mizuno et al. (2006), reported the detection in $\mathrm{CO}(1-0)$ emission of seven new and independent sites in the Bridge out of 16 sites investigated which had correlated bright $\mathrm{H} \mathrm{I}$ emission and $100 \mu \mathrm{m}$ emission. The molecular clouds detected have a weak CO emission (a factor of 10 weaker than that of the SMC), as expected in low mettalicity systems. Mizuno et al. have estimated the molecular mass of the clouds and these range between 1-7 $\times 10^{3} \quad \mathrm{M}_{\odot}$. These molecular clouds lie at a distance of a few tens of parsec of OB associations and $\mathrm{H} \alpha$ emission nebulae. Such evidences support that the molecular clouds in the Bridge could be the sites of new star formation in the Magellanic Bridge. Some peaks of the molecular clouds contain embedded clusters, indicating that star formtion is still going on (see posters by Rubio et al. and Gordon et al. in this volume). Its distance of a few kpc to the SMC wing implies that these have to have formed in situ and could be a prototype of star formation induced in a galaxy-galaxy interaction.

\section{The properties of GMCs}

To study the systematic comparison of GMC properties, individual clouds were identified by the new cloud-finding algorithm, FITSTOPROPS (Rosolowsky \& Leroy 2006). Rosolowsky and Leroy developed a method for minimizing the biases that plague such comparisons. For example, measurement of the cloud radius depends on the sensitivity of the measurements, and they suggest a robust method to extrapolate to the expected radius in the limit of infinite sensitivity. All data (LMC, SMC, the Galaxy) were re-analyzed using the same methods to minimize systematics and cloud properties of GMCs, such as size (radius), line width, CO luminosity, virial mass were derived (for the details, see Fukui et al. 2008). GMC properties measured across the Magellanic Clouds are very much 


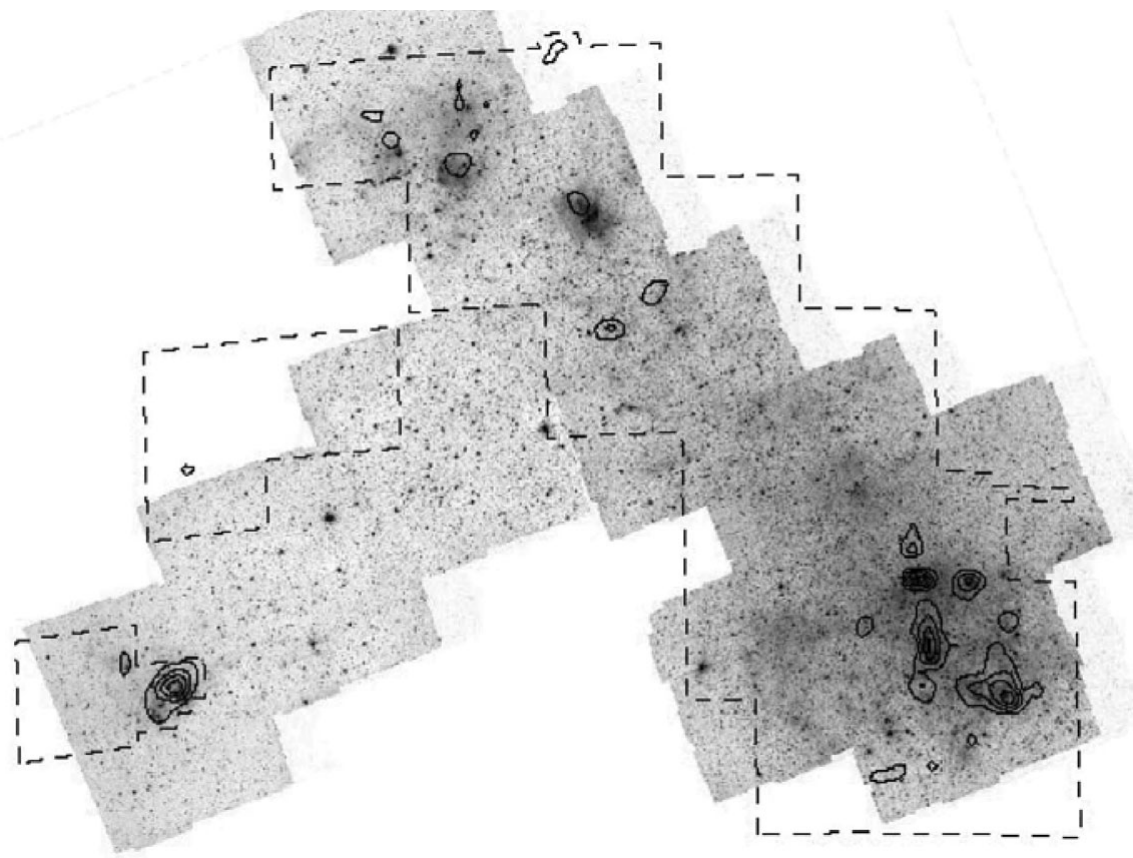

Figure 2. GMCs in the SMC observed with the NANTEN telescope overlayed on an a near-infrared image of the galaxy from the Spitzer Space Telescope (Bolatto et al. 2007). The lines indicate the survey boundary. The CO clouds are clearly associated with regions of transiently heated small grains or PAHs that appear as dark, nebulous regions in the image.

compatible with those in the Galaxy and nearby galaxies (Blitz et al. 2007; Bolatto et al. 2008).

The mass spectrum (Fig. 3, left) of the GMCs in the LMC with $5 \times 10^{4} \leqslant M_{\mathrm{CO}} / \mathrm{M}_{\odot} \leqslant$ $10^{7}$ is well fitted by a power law, $N_{\mathrm{CO}}\left(>M_{\mathrm{CO}}\right) \propto\left(M_{\mathrm{CO}} / \mathrm{M}_{\odot}\right)^{-0.75 \pm 0.06}$. This slope is consistent with the previous results obtained from the Galaxy and nearby galaxies (Fukui et al. 2008; Blitz et al. 2007). The slope of the mass spectrum becomes steeper if we fit only the massive clouds, e.g., $N_{\mathrm{CO}}\left(>M_{\mathrm{CO}}\right) \propto\left(M_{\mathrm{CO}} / \mathrm{M}_{\odot}\right)^{-1.2 \pm 0.2}$ for $M_{\mathrm{CO}} \geqslant 3 \times 10^{5}$ $\mathrm{M}_{\odot}$, which suggests mass truncation. This may suggest that the disruption of the molecular clouds is faster in the massive clouds (Fukui et al. 2001, 2008). It may also suggest that cloud formation takes place inhomogeneously; the mass spectra in different regions of the galaxy may have different slopes and the truncation of the slope might appear when we sum up all the mass spectra within the galaxy. The reason of the truncation is not yet known but the current results present new information leading to a better knowledge of the cloud formation and disruption. A least-squares fit to the virial mass versus CO luminosity relation in the LMC shows a power law, $\log \left(M_{\mathrm{vir}} / \mathrm{M}_{\odot}\right)=26 \log \left[L_{\mathrm{CO}} /\right.$ $\left.\left(\mathrm{K} \mathrm{km} \mathrm{s}^{-1} \mathrm{pc}^{2}\right)\right]^{1.1 \pm 0.3}$, with Spearman rank correlation of 0.8 (Fig. 3, right). This relation suggests that clouds are virialized and the CO luminosity can be a good tracer of mass in the LMC with a quite-constant conversion factor from $L_{\mathrm{CO}}$ to mass throughout the mass range $10^{4}<\left(M_{\mathrm{vir}} / \mathrm{M}_{\odot}\right)<10^{7}$. The average value of $\log \left(M_{\mathrm{vir}} / L_{\mathrm{CO}}\right)$ is $1.2 \pm 0.3$, corresponding to an $X_{\mathrm{CO}}$-factor of $\sim(7 \pm 2) \times 10^{20} \mathrm{~cm}^{-2}\left(\mathrm{~K} \mathrm{~km} \mathrm{~s}^{-1}\right)^{-1}$. The $\mathrm{CO}$ clouds in the SMC and the Warp region lie along the best-fitting power law of GMCs in the LMC. 

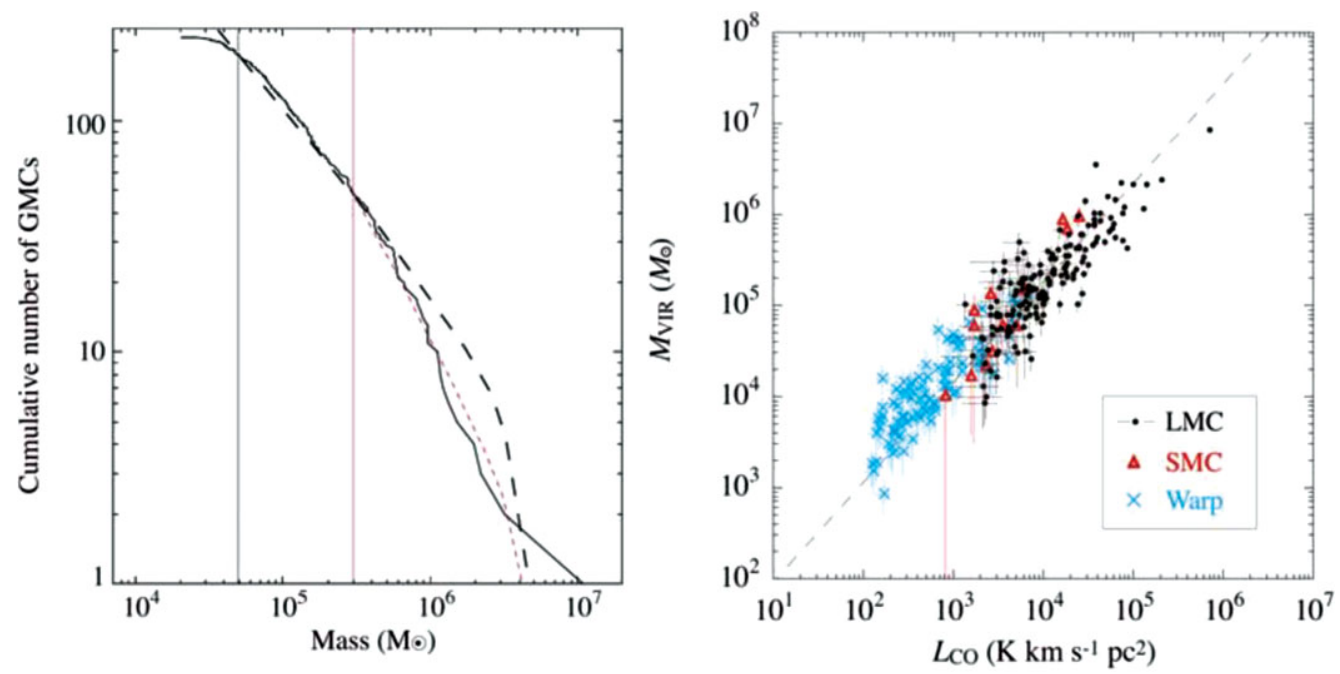

Figure 3. Left: Cumulative mass spectra of the $M_{\mathrm{CO}}$ of the 230 GMCs in the LMC, Right: Virial mass, $M_{\mathrm{vir}}$, of the GMCs as a function of luminosity, $L_{\mathrm{CO}}$. The line presents a best fit to the LMC data (filled circles) with a slope of $1.1 \pm 0.1$. Triangles show the clouds in the SMC (Mizuno et al. 2001b) and crosses those in the Galactic Warp region (Nakagawa et al. 2005).

\section{Star/cluster formation in the GMCs}

The overall distribution of the $\mathrm{H} \alpha$ local peaks and GMCs in the Magellanic Clouds are well coincident to each other, while the extent of the individual $\mathrm{H} \alpha$ emitting regions and the size of the GMCs are different. Almost all the luminous H II regions with high flux densities, like 30 Dor, N 159, N 11 in the LMC and the N 83/84 complex, N 12A, N 24 in the SMC are associated with GMCs, while fainter and diffuse extended $\mathrm{H} \alpha$ emission shows a lower degree of association. Some luminous H II regions, like N 66 in the SMC, are associated with only small molecular clouds, which may indicate molecular gas dissipation after formation of massive $\mathrm{H}$ II regions. It is also noted that there are several GMCs not associated with $\mathrm{H} \alpha$ emission (Fig. 4). Such "starless GMCs" (starless molecular clouds in the sense that they are not associated with $\mathrm{H}$ II regions or young clusters) are apparently very rare in the Solar vicinity (e.g., Maddalena's cloud), where most of the Galactic GMCs are O-star-forming clouds (Fukui et al. 1999; Yamaguchi et al. 2001b). From the comparisons of the LMC GMCs with H II regions and young clusters, a large number of the young clusters $(\tau<10 \mathrm{Myr})$ and $\mathrm{H}$ II regions are found within $100 \mathrm{pc}$ of the GMCs. On the other hand, older clusters show almost no correlation with the GMCs. We have examined the association between the individual GMCs and the H II regions and young clusters and found that about a half of the $\mathrm{H}$ II regions and young clusters are associated with the GMCs.

From the NANTEN CO surveys, three types of GMCs can be classified in terms of star formation activities (Kawamura et al. 2007, 2008); Type I shows no signature of star formation, Type II is associated with relatively small H II region(s) and Type III with both H II region(s) and young stellar cluster(s). It is also found that there is not a significant difference in the distribution of the line widths and sizes of the GMCs among the three types for those with a mass above the completeness limit, $5 \times 10^{4} \mathrm{M}_{\odot}$, while the mass distribution of the Type III GMCs is different from those of Types I and II. The mass distribution of Types I and II shows a peak at $M_{\mathrm{CO}} \sim 10^{5} \mathrm{M}_{\odot}$, while that of the 

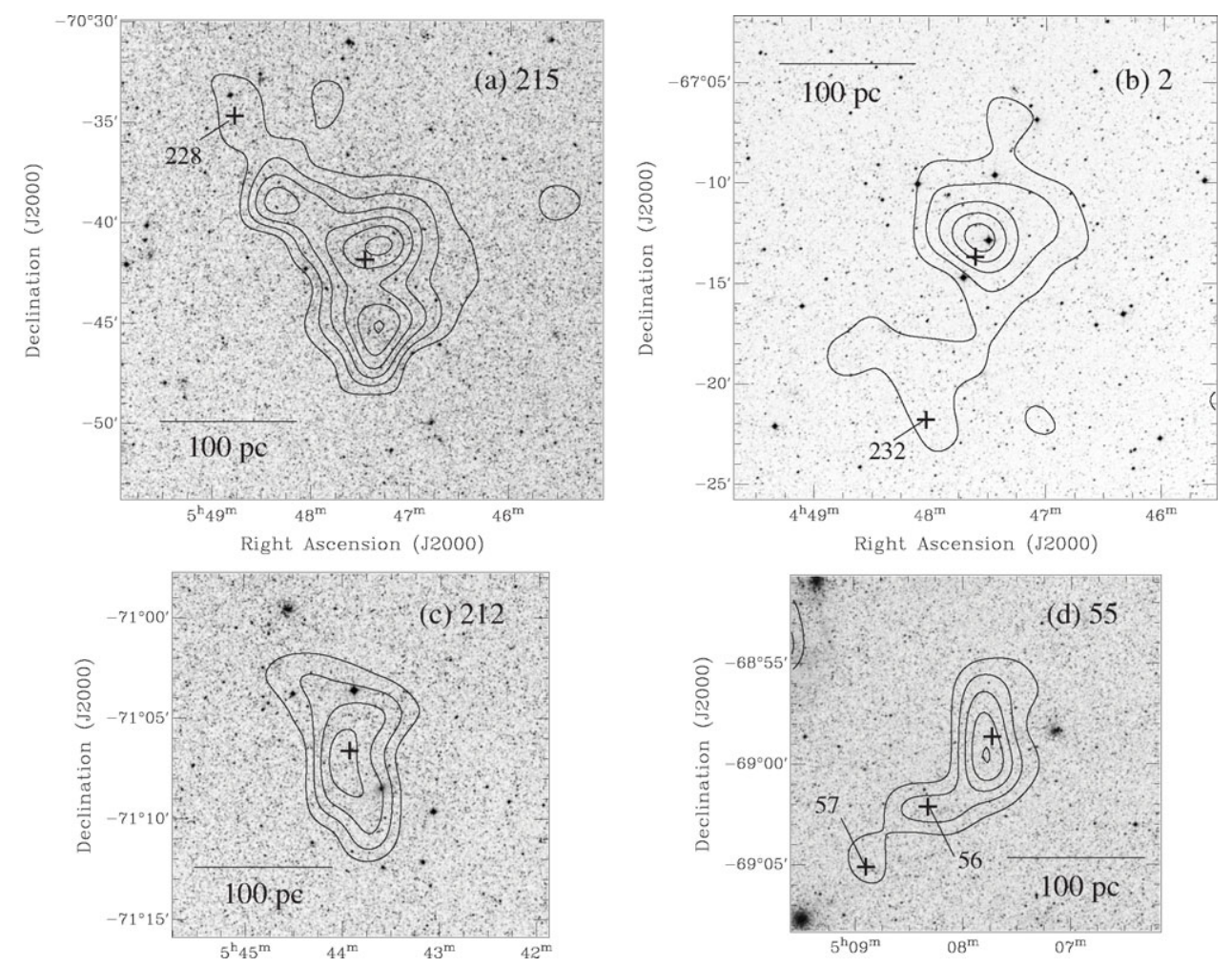

Figure 4. Examples of GMCs without massive star formation (Type I GMCs). NANTEN CO maps are shown in contours superimposed on DSS2 images.

Type III is rather flat. We interpret that these types represent the evolutionary sequence (see Fig. 5 and Kawamura et al. 2007, 2008); i.e. the youngest phase is Type I followed by Type II and the last phase is Type III where most active star formation takes place leading to cloud dispersal. The number of the three types of GMCs should be proportional to the time scale of each evolutionary stage if a steady state is a good approximation. By adopting the time scale of the youngest stellar clusters, $10 \mathrm{Myr}$, we roughly estimate the timescales of Types I, II, and III to be $6 \mathrm{Myr}, 13 \mathrm{Myr}$ and $6 \mathrm{Myr}$, respectively for those with a mass above the completeness limit, $5 \times 10^{4} \mathrm{M}_{\odot}$. This corresponds to a lifetime of the GMCs of 20-30 Myr.

Recent surveys of the Magellanic Clouds by the IR satellites, like Spitzer (e.g., Meixner et al. in this volume, "Surveying the Agents of a Galaxy's Evolution") and AKARI have been strong tools to identify younger, and lower mass YSOs (Whitney et al. 2008). From the comparisons of these YSOs and the GMCs, the distribution of the YSOs appears to be well correlated with that of CO. Significant enhancement of the YSO density is seen toward some of large H II regions, such as 30 Dor, N 11, and N 44 in the LMC. YSO densities towards GMCs with high-mass star formation (Types II and III) are significantly (a factor of two or three) higher than that towards Type I GMCs. This demonstrates that the Type I GMCs have actually lower star formation activity than the others. It also means that the high-mass star formation coincides with the increase of the number of YSOs. 


\section{GMC evolution}
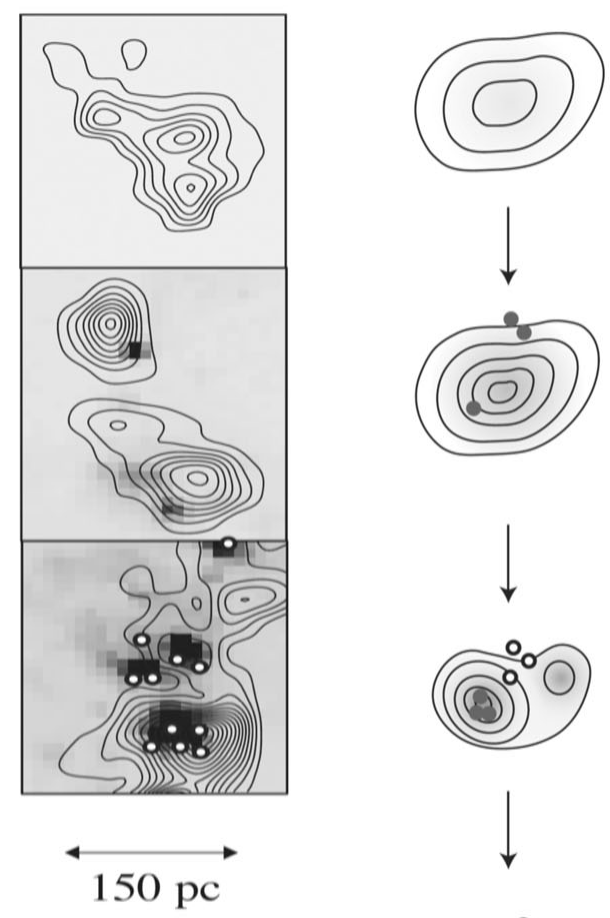

Type I

no massive

star formation

$6 \mathrm{Myr}$

46 clouds

Type II only HII regions

13 Myr

\section{8 clouds}

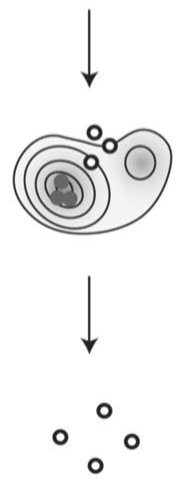

Type III

HII regions and young clusters

$6 \mathrm{Myr}$

\section{7 clouds}

Figure 5. Evolutionary sequence of the GMCs. The left panels are examples of GMC Type I, II, and III from the top panel, respectively. Each panel presents an $\mathrm{H} \alpha$ image from Kim et al. (1999) with GMCs identified by NANTEN (Fukui et al. 2008) in contours. Open circles indicate the positions of young clusters (Bica et al. 1996). The middle panels are illustrations for each evolutionary stage. Open circles and filled circles represent young clusters and H II regions, respectively (Kawamura et al. 2008).

\section{GMCs and $\mathrm{H}$ I gas}

GMCs are the major sites of star formation and the GMC formation and evolution must be a crucial step in the evolution of a galaxy. To examine the relationship of GMCs to the remainder of the neutral ISM, we plot the CO clouds on top of H I maps of the Magellanic Clouds in Fig. 6. Every GMC in each of the galaxies is found on a bright filament or clump of $\mathrm{HI}$, where $N(\mathrm{HI}) \geqslant \sim 10^{21} \mathrm{~cm}^{-2}$, but the reverse is not true: there are many bright filaments of $\mathrm{H}$ I without CO. The Magellanic System comprises H I-rich galaxies, $\left[M(\mathrm{HI}) \sim 5 \times 10^{8} \mathrm{M}_{\odot}(\mathrm{LMC}), 4 \times 10^{8} \mathrm{M}_{\odot}(\mathrm{SMC}), 5 \times 10^{7} \mathrm{M}_{\odot}\right.$ (Bridge) (Brüns et al. 2005). Molecular mass traced by CO, $M_{\mathrm{CO}}$, covers only $\sim 10 \%$ (LMC), $1 \%$ (SMC), $0.05 \%$ (Bridge) of $M(\mathrm{HI})$, respectively, in these galaxies. In the LMC, large $\mathrm{H}$ i integrated intensities and peak brightnesses are prerequisites for detection of $\mathrm{CO}$, with the detection fraction rising steadily for larger values of both quantities, though never reaching $100 \%$. From the 2-D (2 dimensional) analysis (Fig. 7 left), the peak of the histogram is only $33 \%$, so even at the highest H I peak brightnesses, only about $1 / 3^{\text {rd }}$ of the pixels are associated with CO (see poster by Wong et al. this volume). Fukui (2007) made a 3-D (3 dimensional) comparison of $\mathrm{CO}$ and $\mathrm{HI}$ in the LMC where the 3 -D datacube has a velocity axis in addition to the two axes in the sky. Fig. 7 (right) shows a histogram of the $\mathrm{H}$ integrated intensity in 3-D and the pixels with significant $\mathrm{CO}$ emission (greater 

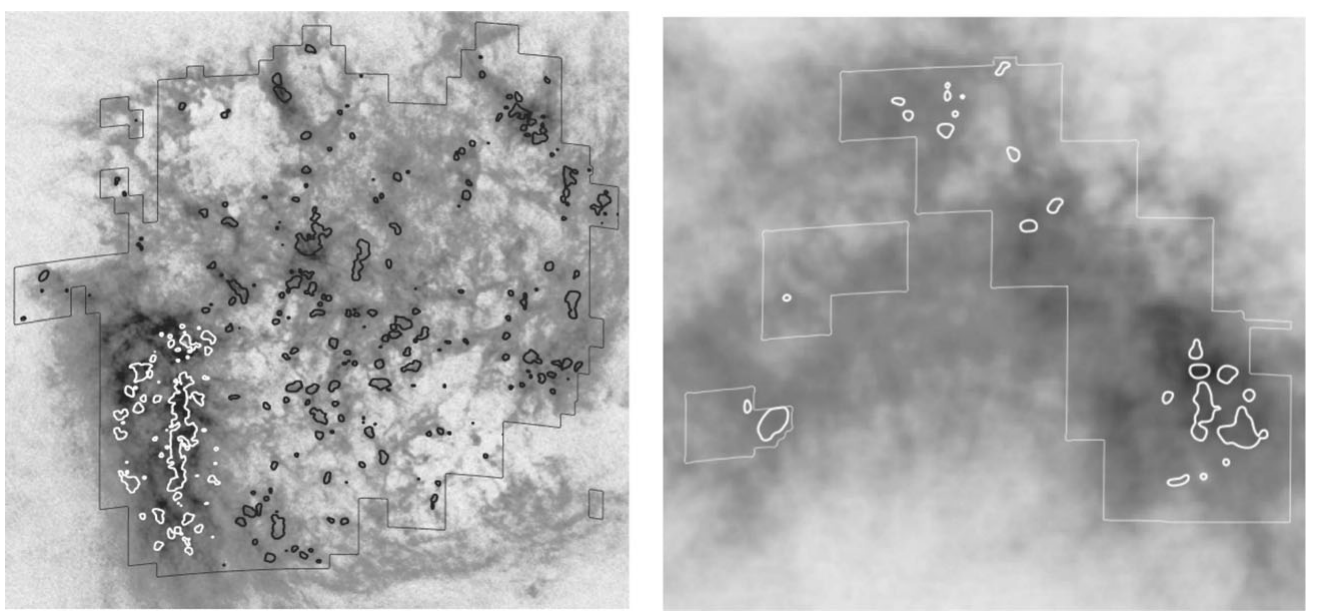

Figure 6. CO emission overlaid on maps of $\mathrm{HI}$ emission for the LMC (left) and the SMC (right). The H I maps are from Kim et al. (2003, LMC) and Stanimirović et al. (1999, SMC). $\mathrm{CO}$ emission (contours) is found exclusively on bright filaments of atomic gas though not every bright $\mathrm{H}$ i filament has $\mathrm{CO}$ emission.
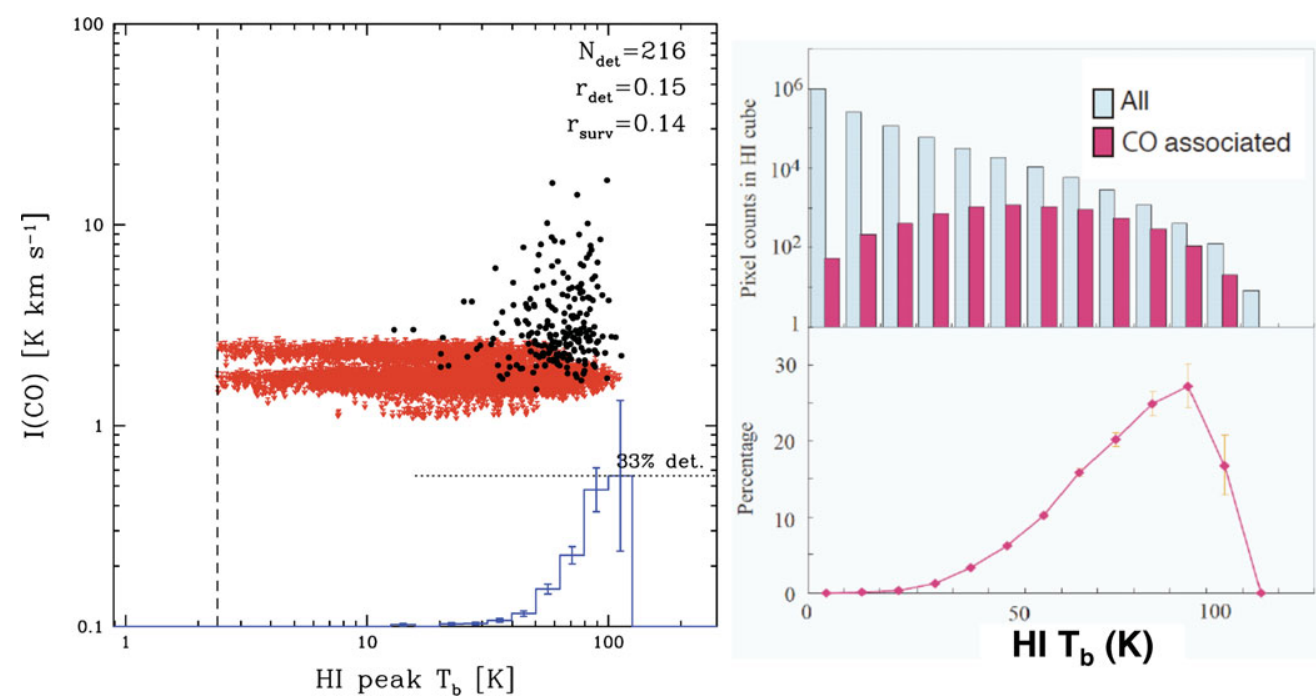

Figure 7. Left: Integrated CO intensity vs. H I intensity (2-D comparisons). Black solid circles are CO detections, red arrows represent 3- $\sigma$ upper limits. Right: (Upper panel) Distribution of $\mathrm{H}$ I integrated intensity in 3-D. Distribution of the $\mathrm{H}$ I integrated intensity where the significant $\mathrm{CO}$ emissions are detected is also shown in gray. (Lower panel) The ratio of the H I pixels with and without $\mathrm{CO}$ emission.

than $0.7 \mathrm{~K} \mathrm{~km} \mathrm{~s}^{-1}$ ) are marked. This histogram shows that the CO fraction increases steadily with the $\mathrm{HI}$ intensity, and about one third of the pixels exhibit $\mathrm{CO}$ emission near $T_{\mathrm{b}}(\mathrm{HI})$ of $\sim 90 \mathrm{~K}$. This is consistent with the 2-D analysis within error. Fig. 8 shows comparisons with $\mathrm{CO}$ and $\mathrm{H}$ i intensity for the three GMC types. This clearly shows that the H I intensity tends to increase from Type I to Type III. The H I intensity surrounding GMCs becomes greater with the GMC evolution and star formation. The dependence of $\mathrm{H}$ I intensity on Types of GMC indicates that GMCs are "dressed" in H I and that the "H I dress" grows in time. The enveloping $\mathrm{H}$ I gas is accreting onto GMCs and is converted into 


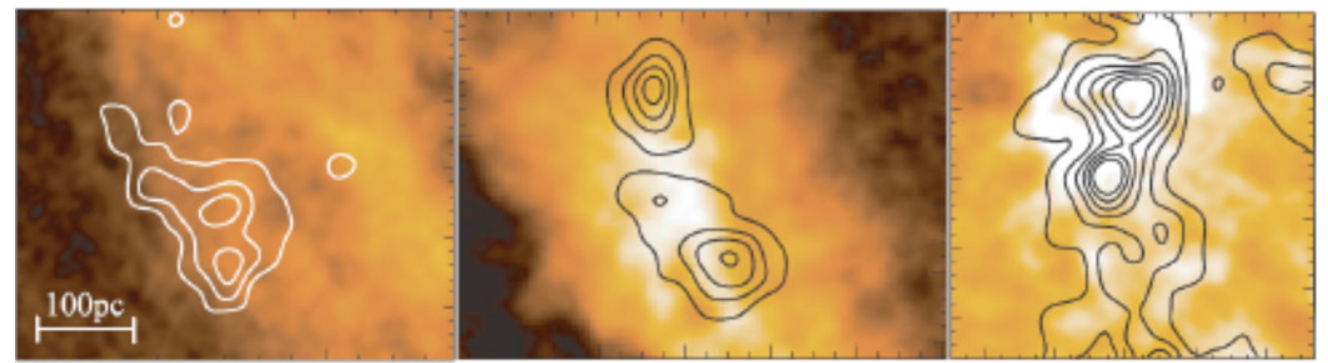

Figure 8. Examples of the H I and CO (contour) distributions of the GMC Type I (Left), II (Middle), and III (Right), respectively. The images are H I integrated intensity maps; velocity is integrated over the range where the significant $\mathrm{CO}$ emissions are detected.

$\mathrm{H}_{2}$ due to increased optical depth. This leads to increase the molecular mass of GMC, i.e. the observed mass increases from Type I to III (see section 5). The timescale of the GMC evolution is $\sim 10 \mathrm{Myr}$ and the increased molecular mass is of the order of $\sim 10^{6}$ $\mathrm{M}_{\odot}$. Hence, a mass accretion rate of $\sim 10^{-1} \mathrm{M}_{\odot} \mathrm{yr}^{-1}$ is required. We roughly estimate that this rate is consistent with that calculated for a spherical accretion of the $\mathrm{HI}$ gas having $n(\mathrm{HI}) \sim 10 \mathrm{~cm}^{-3}$ at an infall speed of $\sim 7 \mathrm{~km} \mathrm{~s}^{-1}$. In the SMC and Bridge, the $\mathrm{H}$ I is so widespread that the $\mathrm{CO}$ clouds appear as small, isolated clouds in a vast sea of $\mathrm{HI}$.

\section{High (sub)millimeter observations of GMCs}

At the resolution of the NANTEN telescope ( $\sim 40 \mathrm{pc})$, many of the star-forming clumps (cores) in the GMCs in the Magellanic System remain unresolved. To more reliably determine their sizes, virial masses, and relationship with star formation and H i structures etc. at 10 pc scale, recently, MAGMA ("The Magellanic Mopra Assessment": see posters by Hughes et al. and Muller et al. in this volume) have started. This new project will cover all the brightest $\mathrm{CO}$ clouds in the LMC and SMC as detected by NANTEN at a resolution of $\sim 45^{\prime \prime}(10 \mathrm{pc})$. Maps are revealing molecular cloud properties across fluxlimited samples in both galaxies. They are also being used to address the relationship with star formation and $\mathrm{CO}-\mathrm{H}$ I correlations, which we are investigating globally using the NANTEN data, can be studied on the scales of individual GMCs.

Recent advances in submillimeter observations have allowed us to determine physical parameters of molecular clouds over much larger ranges than in the millimeter region by comparing line intensities between different transitions. High- $J$ CO and $\mathrm{C}$ I lines are important to constrain physical conditions $\left(n\left(\mathrm{H}_{2}\right), T_{\mathrm{kin}}\right)$, and heating mechanism (UV heating etc.) in clouds (Minamidani et al. in this volume; Minamidani et al. 2008). These submillimeter studies were initiated with the SEST 15-m telescope in Chile followed by the $A S T / R O$ 1.6-m telescope in Antarctica. Subsequently, in the 2000s, the development of new instruments at an altitude of $5000 \mathrm{~m}$ in the Atacama in northern Chile resulted in a superior capability because of the high altitude and dry characteristics of the site. The instruments installed in the Atacama include the ASTE $10 \mathrm{~m}$, APEX 12 $\mathrm{m}$, and NANTEN2 $4 \mathrm{~m}$ telescopes (Fig. 9). All these instruments are beginning to take new molecular data with significantly better quality than before in terms of noise level (Fig. 10), as well as angular resolution (Fig. 11). It is also noteworthy that the current frequency coverage extends as high as $800 \mathrm{GHz}$.

The N 159 region is one of the most interesting star/cluster-forming regions in the LMC. This region has been well studied at $\mathrm{mm} / \mathrm{submm}$ and infrared wavelengths (see poster by Y. Mizuno et al. in this volume). It has been shown that the N 159 complex 

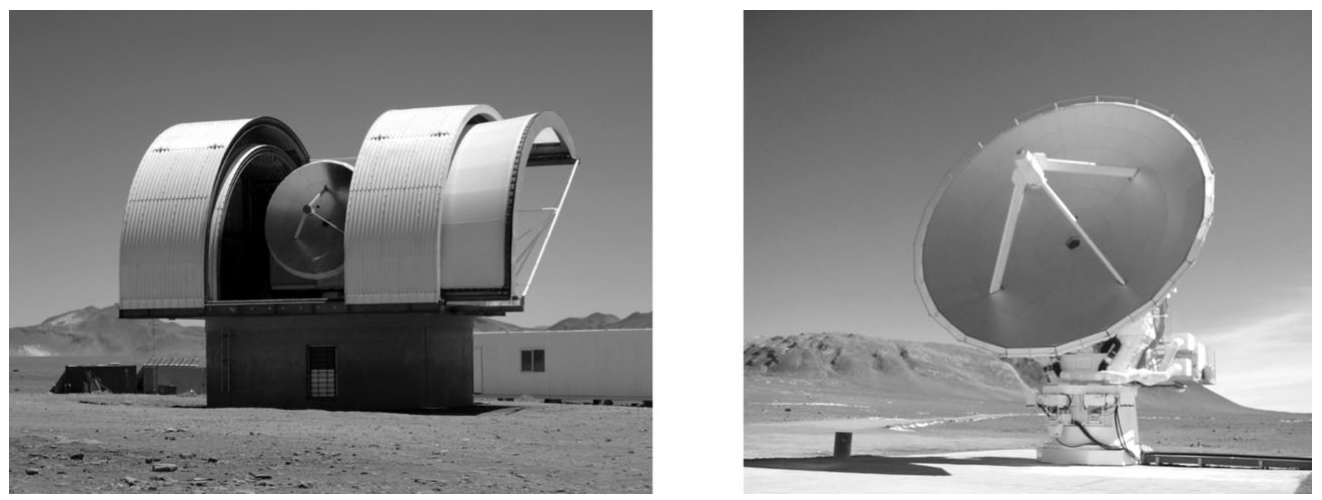

Figure 9. NANTEN2 (left) and ASTE (right) telescopes.

consists of 3 distinct and spatially well separated clumps (Fig. 11). N 159W is a prominent star-forming region and has the highest observed $\mathrm{CO}(J=1-0,2-1,3-2)$ intensities in the LMC. This clump is interesting to study the origin of young massive (populous) clusters. On the contrary, the southern clump, N 159S, lacks signs of on-going massive star formation (neither emission nebulae nor clusters) and appears to be a cold, more quiescent cloud. This clump is a good candidate of a chemically young GMC and crucial for the origin of starless GMCs.

For the SMC, submillimeter observations of $\mathrm{CO}(4-3 / 7-6)$ and $[\mathrm{CI}$ ] lines towards the star forming regions are ongoing with NANTEN2. From the LVG model calculations, we derived the densities and temperatures of the dense clumps in the GMCs, which show a good correlation with the star formation activities in the GMCs. The GMC associated with N 66 is hot and dense, on the other hand, the GMC associated SMC B1\#1-1, which is a quiescent GMC devoid of any massive star forming activity, shows up cold and less dense. The relation between these properties and the evolutionary sequence of GMCs are discussed in the poster by N. Mizuno et al. (this volume).

The current study of GMCs in the Magellanic System provides us with promise on what we can learn on GMCs and star formation therein when $A L M A$ is used for the Local Group. It would be particularly important to find very high mass cloud cores of $\sim 10^{5}-10^{6} \mathrm{M}_{\odot}$, as promising candidates for proto-globular clusters.

\section{Acknowledgements}

NANTEN2 is an international collaboration between 10 universities including the Universities of Cologne and Bonn. The original NANTEN telescope was operated based on a mutual agreement between Nagoya University and the Carnegie Institution of Washington. We also acknowledge that the operation of NANTEN was realized by contributions from many Japanese public donators and companies. This work is financially supported in part by a Grant-in-Aid for Scientific Research (KAKENHI) from the Ministry of Education, Culture, Sports, Science and Technology of Japan (Nos. 15071203 and 18026004) and from JSPS (Nos. 14102003, 20244014, and 18684003). This work is also financially supported in part by core-to-core program of a Grant-in-Aid for Scientific Research from the Ministry of Education, Culture, Sports, Science and Technology of Japan (No. 17004). It is a pleasure to thank my collaborators, Nagoya University (A. Kawamura, Y. Mizuno, E. Muller, T. Onishi, M. Murai, A. Ohama, H. Yamamoto, A. Mizuno, Y. Fukui), the NANTEN2 consortium (M. Rubio, J. Pineda, F. Bertoldi, U. Klein, J. Stutzki, Y. Yonekura), the ASTE team (T. Minamidani, N. Yamaguchi, M. Ikeda, K. Tatematsu, T. Hasegawa), the MAGMA project (T. Wong, A. Hughes, J. Pineda, J. Ott, 
N159W

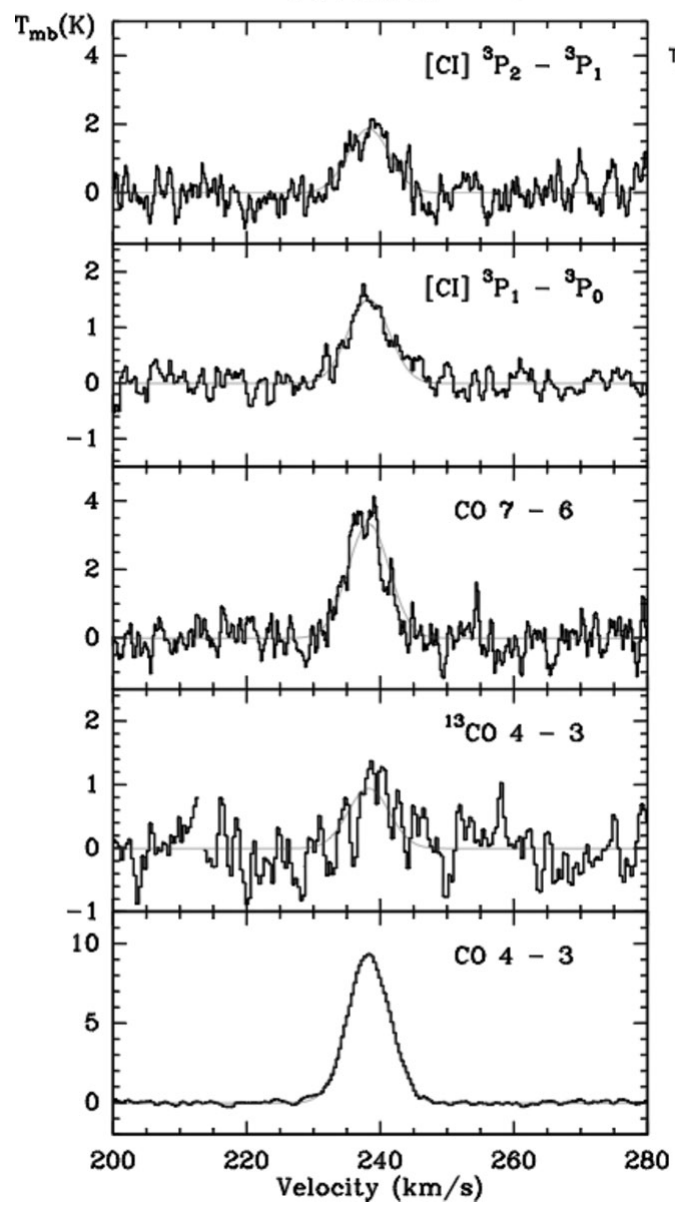

30Dor-10

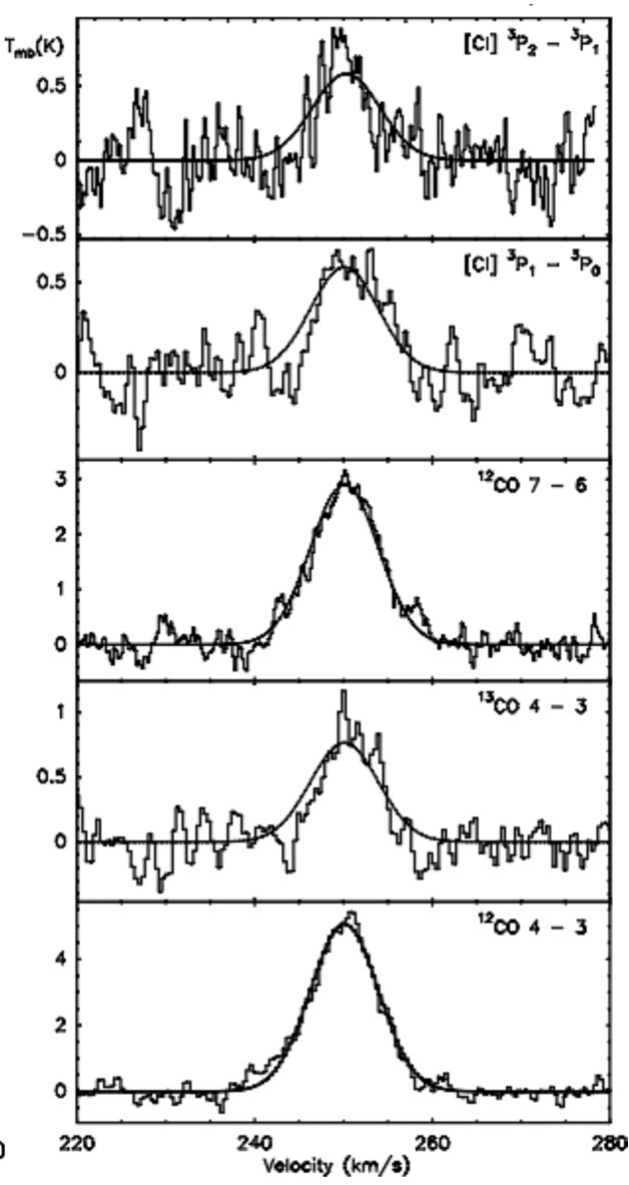

Figure 10. Submm spectra towards N 159W (left) and 30 Dor-10 (right) taken with NANTEN2 (Pineda et al. 2008).

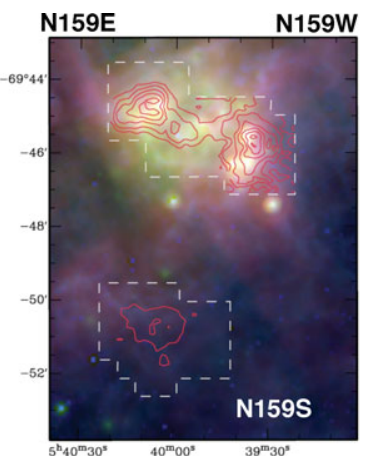

Spitzer $(8,24 \mu \mathrm{m})$ (Meixner et al.2006) +NANTEN2 CO (4-3)

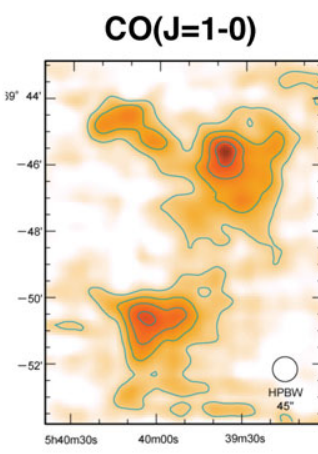

Mopra

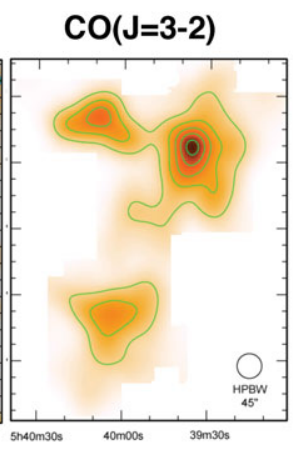

ASTE

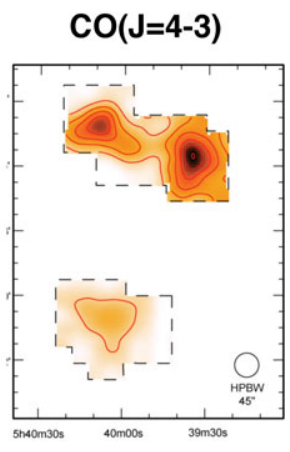

NANTEN2

Figure 11. Integrated intensity maps of $\mathrm{CO}(J=1-0 / 3-2 / 4-3)$ towards the $\mathrm{N} 159$ region in the LMC. the resolutions of these maps were smoothed to the same beam size of $45^{\prime \prime}$. 
et al.), the SEST Nagoya-Onsala project (L. E. B. Johansson, R. S. Booth, S. Nikolić, A. Heikkila, L.-Å. Nyman, M. Lerner), ATNF users (S. Kim, Staveley-Smith, M. D. Filipović), and the Spitzer SAGE Team.

\section{References}

Bica, E., Clariá, J. J., Dottori, H., Santos, J. F. C., Jr., \& Piatti, A. E. 1996, ApJS, 102, 57

Blitz, L., Fukui, Y., Kawamura, A., Leroy, A., Mizuno, N., \& Rosolowsky, E. 2007, in B. Reipurth, D. Jewitt, \& K. Keil (eds.), Protostars and Planets $V$ (Tucson: University of Arizona Press), p. 951

Bolatto, A. D., Simon, J. D., Stanimirović, S., et al. 2007, ApJ, 655, 212

Bolatto, A. D., Leroy, A. K., Rosolowsky, E., Walter, F., \& Blitz, L. 2008, ApJ, 686, 948

Bot, C., Boulanger, F., Rubio, M., \& Rantakyro, F. 2007, A\&A, 471, 103

Brüns, C., Kerp, J., Staveley-Smith, L., et al. 2005, A\&̈A, 432, 45

Cohen, R. S., Dame, T. M., Garay, G., Montani, J., Rubio, M., \& Thaddeus, P. 1988, ApJ, 331, L95

de Boer, K. S., Braun, J. M., Vallenari, A., \& Mebold, U. 1998, A\&̈A, 329, 49

Fukui, Y., Mizuno, N., Yamaguchi, R., et al. 1999, PASJ, 51, 745

Fukui, Y., Mizuno, N., Yamaguchi, R., Mizuno, A., \& Onishi, T. 2001, PASJ, 53, L41

Fukui, Y. 2007, in B. G. Elmegreen \& J. Palouš (eds.), Triggered Star Formation in a Turbulent ISM, IAU Conf. Proc. (Cambridge: CUP), p. 31

Fukui, Y., Kawamura, A., Minamidani, T., et al. 2008, ApJS, 178, 56

Fujimoto, M. \& Noguchi, M. 1990, PASJ, 42, 505

Kawamura, A., Minamidani, T., Mizuno, Y., Onishi, T., Mizuno, N., Mizuno, A., \& Fukui, Y. 2007, in B. G. Elmegreen \& J. Palouš (eds.), Triggered Star Formation in a Turbulent ISM, IAU Conf. Proc. (Cambridge: CUP), p. 101

Kawamura, A., Minamidani, T., Mizuno, Y., Onishi, T., Mizuno, N., Mizuno, A., \& Fukui, Y. 2008, ApJS, submitted

Kim, S., Staveley-Smith, L., Dopita, M. A., Freeman, K. C., Sault, R. J., Kesteven, M. J., \& McConnell, D. 1998, ApJ, 503, 674

Kim, S., Dopita, M. A., Staveley-Smith, L., \& Bessell, M. S. 1999, AJ, 118, 2797

Kim, S., Staveley-Smith, L., Dopita, M. A., Sault, R. J., Freeman, K. C., Lee, Y., \& Chu, Y.-H. 2003, ApJS, 148, 473

Kutner, M. L., Rubio, M., Booth, R. S., et al. 1997, A\&SAS, 122, 255

Leroy, A., Bolatto, A. D., Stanimirović, S., Mizuno, N., Israel, F., \& Bot, C. 2007, ApJ, 658, 1027

Minamidani, T., Mizuno, N., Mizuno, Y., et al. 2008, ApJS, 175, 485

Mizuno, N., Yamaguchi, R., Mizuno, A., et al. 2001a, PASJ, 53, 971

Mizuno, N., Rubio, M., Mizuno, A., Yamaguchi, R., Onishi, T., \& Fukui, Y. 2001b, PASJ, 53, L45

Mizuno, N., Muller, E., Maeda, H., Kawamura, A., Minamidani, T., Onishi, T., Mizuno, A., \& Fukui, Y. 2006, ApJ, 643, L107

Muller, E., Staveley-Smith, L., \& Zealey, W. J. 2003, MNRAS, 338, 609

Nakagawa, M., Onishi, T., Mizuno, A., \& Fukui, Y. 2005, PASJ, 57, 917

Ott J., Wong, T., Pineda, J., et al. 2008, PASA, 25, 129

Pineda, J. L., Mizuno, N., Stutzki, J., et al. 2008, A\&A, 482, 197

Rosolowsky, E. \& Leroy, A. 2006, PASP, 118, 590

Rubio, M., Lequeux, J., \& Boulanger, F. 1993, A\&SA, 271, 9

Rubio, M., Boulanger, F., Rantakyro, F., \& Contursi, A 2004, A\&A, 425, L1

Stanimirović, S., Staveley-Smith, L., Dickey, J. M., Sault, R. J., \& Snowden, S. L. 1999, MNRAS, 302,417

Whitney, B. A., Sewilo, M., Indebetouw, R., et al. 2008, AJ,553, 185

Yamaguchi, R., Mizuno, N., Onishi, T., Mizuno, A., \& Fukui, Y. 2001a, ApJ, 553, L185

Yamaguchi R., Mizuno, N., Onishi, T., Mizuno, A., \& Fukui, Y. 2001b, PASJ 53, 959

Yamaguchi R., Mizuno, N., Mizuno, A., et al. 2001c, PASJ 53, 985 